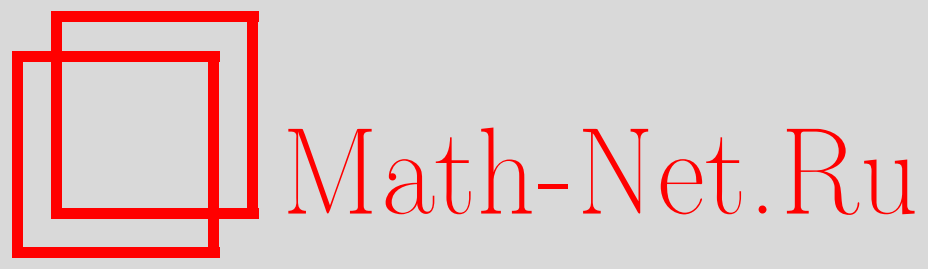

Юн Цзяо, Тао Ма, Пэйдэ Лю, О вложениях пространств Лоренца векторнозначных мартингалов, Функи. анализ и его прил., 2010, том 44, выпуск $3,92-96$

DOI: https://doi.org/10.4213/faa3001

Использование Общероссийского математического портала MathNet.Ru подразумевает, что вы прочитали и согласны с пользовательским соглашением

http://www.mathnet.ru/rus/agreement

Параметры загрузки:

IP : 54.89 .56 .158

26 апреля 2023 г., 16:07:25

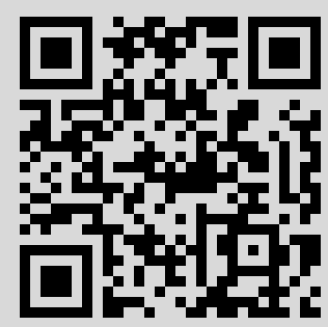


УДК 517.9

\title{
О вложениях пространств Лоренца векторнозначных мартингалов*
}

\author{
(c) 2010. Юн Цзяо, ТАО МА, Пэйдэ Лю
}

1. Введение. Известно, что справедливость классических (скалярных) результатов для случая функций или мартингалов со значениями в банаховом пространстве зависит от геометрических свойств этого пространства. Как правило, речь идет о равномерной выпуклости или гладкости. Например, замечательный результат Пизье [7] состоит в следующем. Пусть $1<q<\infty$. Тогда банахово пространство $X$ имеет эквивалентную $q$-равномерно выпуклую норму в том и только том случае, когда для некоторого (или, эквивалентно, для каждого) $p, 1<p<\infty$, существует положительная постоянная $C$, такая, что

$$
\left\|\left(\left\|f_{1}\right\|^{q}+\sum_{n \geqslant 2}\left\|f_{n}-f_{n-1}\right\|^{q}\right)^{1 / q}\right\|_{p} \leqslant C \sup _{n \geqslant 1}\left\|f_{n}\right\|_{p}
$$

для всех $L_{p}$-мартингалов $f$ со значениями в пространстве $X$. Справедливость же обратного неравенства позволяет утверждать, что на $X$ существует эквивалентная $q$-равномерно гладкая норма. Основная цель настоящей работы состоит в том, чтобы установить аналог неравенства (1) для мартингалов $f$, являющихся элементами пространства Лоренца. Следует отметить, что в данной работе применяются мартингальные преобразования, заданные последовательностями операторов, которые были введены в [6]. Такой подход дает краткий и ясный способ получения мартингальных неравенств рассматриваемого вида.

\section{2. Предварительные сведения и вспомогательные утверждения.} Пусть $(\Omega, \mathscr{F}, \mathrm{P})$ - полное вероятностное пространство и $f-$ случайная величина на нем со значениями в $X$. Пространство Лоренца $L^{p, q}(\Omega ; X)=L^{p, q}$, $0<p<\infty, 0<q \leqslant \infty$, состоит из всех измеримых функций $f$, для которых полунорма $\|f\|_{p, q}$, заданная формулами

$$
\begin{gathered}
\|f\|_{p, q}=\left(\frac{q}{p} \int_{0}^{1}\left(t^{1 / p} f^{*}(t)\right)^{q} \frac{d t}{t}\right)^{1 / q}, \quad 0<q<\infty, \\
\|f\|_{p, \infty}=\sup _{t>0} t^{1 / p} f^{*}(t),
\end{gathered}
$$

конечна. Здесь $f^{*}(t)=\inf \{s>0: \mathrm{P}(\|f\|>s) \leqslant t\}, t \in[0,1]$, обозначает убывающую перестановку функции $f$. Более подробно о пространствах Лоренца см. в [1].

Пусть $X$ - банахово пространство, $f=\left(f_{n}\right)_{n \geqslant 1}$ - мартингал со значениями в $X$ и $d f=\left(d f_{n}\right)_{n \geqslant 1}$ - мартингал-разность для $f, d f_{n}=f_{n}-f_{n-1}$. Будем использовать следующие обозначения, принятые в теории мартингалов:

*Работа выполнена при поддержке Agence Nationale de Recherche (06-BLAN-0015), National Natural Foundation of China (10371093) 


$$
\begin{gathered}
M_{n}(f)=\sup _{1 \leqslant k \leqslant n}\left\|f_{k}\right\|, \quad M(f)=\sup _{n \geqslant 1}\left\|f_{n}\right\|, \\
S_{n}^{(q)}(f)=\left(\sum_{k=1}^{n}\left\|d f_{k}\right\|^{q}\right)^{1 / q}, \quad S^{(q)}(f)=\left(\sum_{k=1}^{\infty}\left\|d f_{k}\right\|^{q}\right)^{1 / q} .
\end{gathered}
$$

Для $r, 1<r<\infty$, и $s, 1 \leqslant s \leqslant \infty$, определим следующие пространства мартингалов:

$$
\begin{aligned}
H_{r, s}^{*} & =\left\{f=\left(f_{n}\right)_{n \geqslant 1}:\|M(f)\|_{r, s}<\infty\right\}, \\
{ }_{q} H_{r, s}^{S} & =\left\{f=\left(f_{n}\right)_{n \geqslant 1}:\left\|S^{(q)}(f)\right\|_{r, s}<\infty\right\} .
\end{aligned}
$$

Для $X$-значного мартингала $f=\left(f_{n}\right)_{n \geqslant 1}$ положим

$$
\|f\|_{p, q}=\sup _{n}\left\|f_{n}\right\|_{p, q} \text {. }
$$

В [8], [9] подробно изложена скалярная теория мартингалов, а в [2], [4] теория векторнозначных мартингалов.

Определение 1. Пусть $X_{1}$ и $X_{2}$ - банаховы пространства и $L\left(X_{1}, X_{2}\right)$ пространство линейных ограниченных операторов, действующих из $X_{1}$ в $X_{2}$. Определим оператор $T$ следующим образом. Пусть $v=\left\{v_{n}\right\}_{n \geqslant 1}$ - последовательность $L\left(X_{1}, X_{2}\right)$-значных отображений, определенных на $(\Omega, \mathscr{F}, \mathrm{P})$, такая, что $v_{n} \in L_{\infty}\left(L\left(X_{1}, X_{2}\right)\right)$ и $\sup _{n \geqslant 1}\left\|v_{n}\right\|_{L_{\infty}\left(L\left(X_{1}, X_{2}\right)\right)} \leqslant 1$. Для каждого $X_{1}$-значного мартингала $f=\left(f_{n}\right)_{n \geqslant 1}$ положим

$$
(T f)_{n}=\sum_{k=1}^{n} v_{k} d f_{k}
$$

Тогда $T f$ называется мартингальным преобразованием мартингала $f$, задаваемым последовательностью $v$, а оператор $T$ - оператором мартингального преобразования.

Используя разложение Дэвиса, можно установить справедливость следующего утверждения (см., например, [5]).

Лемма 2. Пусть $1<p<\infty, 1 \leqslant q \leqslant \infty$. Тогда каждый $X$-значный мартингал $f=\left(f_{n}\right)_{n \geqslant 1} \in H_{p, q}^{*}$ можсно представить в виде суммы $f=g+h$, где $g$ u $\mathrm{h}$ - мартингаль, удовлетворяющие следующим условиям:

(1) $\left\|d g_{n}\right\| \leqslant 4 M_{n-1}(d f)$;

(2) $\left\|\sum_{n=1}^{\infty}\right\| d h_{n}\|\|_{p, q} \leqslant C\|M(d f)\|_{p, q}$.

Лемма 3. Пусть $X_{1}$ и $X_{2}$ - банаховы пространства, а $T$ - определенный выше оператор мартингального преобразования. Тогда следующие утверждения эквивалентны:

(1) существует положительная постоянная $C$, такая, что $\lambda \mathrm{P}(M(T f)>\lambda)$ $\leqslant C\|M(f)\|_{1}$ для любого $\lambda>0$;

(2) для любъх $p, q, 1<p<\infty, 1 \leqslant q \leqslant \infty$, существует постоянная $C=$ $C_{p, q}>0$, такая, что $\|M(T f)\|_{p, q} \leqslant C\|M(f)\|_{p, q}$;

(3) для некоторых $p_{0}, q_{0}, 1<p_{0}<\infty, 1 \leqslant q_{0} \leqslant \infty$, существует постоянная $C=C_{p_{0}, q_{0}}>0$, такая, что $\|M(T f)\|_{p_{0}, q_{0}} \leqslant C\|M(f)\|_{p_{0}, q_{0}}$.

Доказательство. $(1) \Rightarrow(2)$. Рассмотрим мартингал $f \in H_{p, q}^{*}$. Согласно лемме 2, мы можем представить $f$ в виде $f=g+h$. Тогда

$$
\|M(T f)\|_{p, q} \leqslant C\|M(T g)\|_{p, q}+C\|M(T h)\|_{p, q} .
$$


В силу ограниченности последовательности $\left(v_{k}\right)$ получаем, что

$$
\|M(T h)\|_{p, q} \leqslant\left\|\sup _{n} \sum_{k=1}^{n}\right\| d h_{k}\left\|_{X_{1}}\right\|_{p, q}=\left\|\sum_{k=1}^{n}\right\| d h_{k}\left\|_{X_{1}}\right\|_{p, q} \leqslant C\|M(f)\|_{p, q} .
$$

Положим $W_{n}=4 M_{n-1}(d f)$. Тогда последовательность $\left(W_{n}\right)$ является неубывающей, причем каждая случайная величина $W_{n} \mathscr{F}_{n-1}$-измерима. Зафиксируем $\lambda>0$. Для $\beta>0, \delta>0$, таких, что $\beta>\delta+1$, определим моменты остановки

$$
\begin{gathered}
\mu=\inf \left\{n:\left\|(T g)_{n}\right\|_{X_{2}}>\lambda\right\}, \quad \nu=\inf \left\{n:\left\|(T g)_{n}\right\|_{X_{2}}>\beta \lambda\right\}, \\
\sigma=\inf \left\{n: \max \left\{\left\|g_{n}\right\|_{X_{1}}, W_{n+1}\right\}>\delta \lambda\right\} .
\end{gathered}
$$

Положим $u_{n}=\mathbf{1}_{\{\mu<n \leqslant \min \{\nu, \sigma\}\}}$. Так как $\{\mu<n \leqslant \min \{\nu, \sigma\}\} \in \mathscr{F}_{n-1}$, мы можем рассмотреть мартингал $a=\left(a_{n}\right), a_{n}=\sum_{k=1}^{n} u_{k} d g_{k}$, и его мартингальное преобразование $(\mathrm{Ta})_{n}=\sum_{k=1}^{n} v_{k} u_{k} d g_{k}$. Заметим, что $\left\|d g_{n}\right\| \leqslant W_{n}$. По определению момента остановки $\sigma$ имеем $M(a) \leqslant 2 \delta \lambda$ на множестве $\{\mu<\infty\}$ и $M(a)=0$ на множестве $\{\mu=\infty\}$. Тогда

$$
\|M(a)\|_{1} \leqslant 2 \delta \lambda \mathrm{P}(\mu<\infty)=2 \delta \lambda \mathrm{P}(M(T g)>\lambda) .
$$

Согласно условию (1), получаем, что

$$
\mathrm{P}(M(T a)>(\beta-\delta-1) \lambda) \leqslant \frac{C\|M(a)\|_{1}}{(\beta-\delta-1) \lambda} \leqslant \frac{2 C \delta}{\beta-\delta-1} \mathrm{P}(M(T g)>\lambda) .
$$

Если $w \in\{\mu<n \leqslant \min \{\nu, \sigma\}\}$, то $(T a)_{n}=(T g)_{n} ;$ легко видеть, что

$$
\mathrm{P}(M(T g)>\beta \lambda, M(W) \leqslant \delta \lambda) \leqslant \mathrm{P}(M(T a)>(\beta-\delta-1) \lambda) .
$$

Таким образом,

$$
\begin{aligned}
\mathrm{P}(M(T g)>\beta \lambda) & \leqslant \mathrm{P}(M(T g)<\beta \lambda, M(W) \leqslant \delta \lambda)+\mathrm{P}(M(W)>\delta \lambda) \\
& \leqslant \frac{2 C \delta}{\beta-\delta-1} \mathrm{P}(M(T g)>\lambda)+\mathrm{P}(M(W)>\delta \lambda) .
\end{aligned}
$$

Положим $\rho=2 C \delta /(\beta-\delta-1)$. Согласно эквивалентному определению нормы в $L^{p, q}$, получаем, что

$$
\begin{aligned}
\beta^{-1}\|M(T g)\|_{p, q} & \leqslant \rho^{1 / p}\|M(T g)\|_{p, q}+\delta^{-1}\|M(W)\|_{p, q} \\
& \leqslant \rho^{1 / p}\|M(T g)\|_{p, q}+8 \delta^{-1}\|M(f)\|_{p, q} .
\end{aligned}
$$

Возьмем теперь $\delta$, такое, что $1-\beta \rho^{1 / p}>0$. Тогда $\|M(T g)\|_{p, q} \leqslant C\|M(f)\|_{p, q}$. Наконец, из (2) следует, что $\|M(T f)\|_{p, q} \leqslant C\|M(f)\|_{p, q}$. Импликация $(2) \Rightarrow(3)$ очевидна, а $(3) \Rightarrow(1)$ можно доказать, используя не разложение Дэвиса, как выше в лемме 2, а разложение Ганди [5].

3. Вложение мартингальных пространств Лоренца. Выбор двух конкретных последовательностей $v=\left(v_{n}\right)$ операторов позволяет рассматривать $M(f)$ и $S^{(q)}(f)$ как операторы мартингальных преобразований, что вместе с леммой 3 дает прямой способ получить некоторые вложения пространств Лоренца векторнозначных мартингалов.

Теорема 4. Пусть $X$ - банахово пространство. Для любого $q, 2 \leqslant q<\infty$, следующие утверждения эквивалентны:

(1) X изоморфно q-равномерно выпуклому пространству; 
(2) существует константа $C>0$, такая, что для любого $X$-значного мартингала $f$

$$
\lambda \mathrm{P}\left(S^{(q)}(f)>\lambda\right) \leqslant C\|M(f)\|_{1} \quad \forall \lambda>0 .
$$

(3) для любъх (или, эквивалентно, для некоторых) $r, s, 1<r<\infty, 1 \leqslant s \leqslant$ $\infty$,

$$
H_{r, s}^{*} \hookrightarrow q H_{r, s}^{S} .
$$

Доказательство. Эквивалентность (1) $\Leftrightarrow(2)$ доказана в [4]. Рассмотрим следующий оператор $T$, переводящий семейство $X$-значных мартингалов в семейство $\ell^{q}(X)$-значных мартингалов. Пусть $v_{k} \in L\left(X, \ell^{q}(X)\right)$ - оператор, определяемый равенством $v_{k} x=\left\{x_{j}\right\}_{j=1}^{\infty}$ для $x \in X$, где $x_{j}=x$, если $j=k$, и $x_{j}=0$ в противном случае. Пусть

$$
(T f)_{n}=\sum_{k=1}^{n} v_{k} d f_{k}=\left(d f_{1}, d f_{2}, \ldots, d f_{n}, 0, \ldots\right)
$$

Тогда $M(T f)=\sup _{n}\left\|(T f)_{n}\right\|_{\ell^{q}(X)}=S^{(q)}(f)$.

Эквивалентность $(1) \Leftrightarrow(2)$ гарантирует, что оператор $T$ удовлетворяет условию (1) леммы 3. Отсюда сразу следует требуемая эквивалентность $(1) \Leftrightarrow(3)$.

Теорема 5. Пусть $X-$ банахово пространство. Для $1<q \leqslant 2$ следующие утверждения эквивалентны:

(1) X изоморфно q-равномерно гладкому пространству;

(2) существует константа $C>0$, такая, что для любого $X$-значного мартингала $f$

$$
\lambda \mathrm{P}(M(f)>\lambda) \leqslant C\left\|S^{(q)}(f)\right\|_{1} \quad \forall \lambda>0 ;
$$

(3) для любых (или, эквивалентно, для некоторых) $r, s, 1<r<\infty, 1 \leqslant s \leqslant$ $\infty$

$$
{ }_{q} H_{r, s}^{S} \hookrightarrow H_{r, s}^{*} .
$$

Доказательство. Эквивалентность (1) $\Leftrightarrow(2)$ следует из [4]. Рассмотрим $\ell^{q}(X)$-значные мартингалы $F=\left(F_{n}\right)_{n \geqslant 1}, F_{n}=\sum_{k=1}^{n} D_{k}, D_{k}=\left(D_{k}^{j}\right)_{j \geqslant 1}$, и следующий оператор мартингального преобразования $R$, действующий из семейства $\ell^{q}(X)$-значных мартингалов в семейство $X$-значных мартингалов. Пусть $v_{k} \in L\left(\ell^{q}(X), X\right)$ - оператор, определяемый равенством $v_{k} x=x^{k}$ для $x=$ $\left\{x^{j}\right\}_{j \geqslant 1} \in \ell^{q}(X)$, и пусть

$$
(R F)_{n}=\sum_{k=1}^{n} v_{k} D_{k}=\sum_{k=1}^{n} v_{k} D_{k}^{k}
$$

Теперь для любого $X$-значного мартингала $f$ с $f_{n}=\sum_{k=1}^{n} d f_{k}$ мы можем выбрать $\ell^{q}(X)$-значный мартингал $F=\left(F_{n}\right)_{n \geqslant 1}$ с $D_{k}^{j}=d f_{k}$, если $j=k$, и $D_{k}^{j}=0$, если $j \neq k$. Тогда

$$
(R F)_{n}=\sum_{k=1}^{n} D_{k}^{k}=\sum_{k=1}^{n} d f_{k}=f_{n}, \quad M(R F)=M(f),
$$

и

$$
\left\|F_{n}\right\|_{\ell^{q}(X)}=\left\|\left(d f_{1}, d f_{2}, \ldots, d f_{n}, 0, \ldots\right)\right\|_{\ell^{q}(X)}=S_{n}^{(q)}(f), \quad M(F)=S^{(q)}(f) .
$$


Эквивалентность (1) $\Leftrightarrow(2)$ гарантирует, что оператор мартингального преобразования $R$ удовлетворяет условию (1) леммы 3 . Отсюда следует доказываемая эквивалентность $(1) \Leftrightarrow(3)$.

Следствие 6. Пусть $X$ - банахово пространство. Следующие утверждения эквивалентны:

(1) X изоморфно гилъбертову пространству;

(2) для любых (или, эквивалентно, для некоторых) $r, s, 1<r<\infty, 1 \leqslant s \leqslant$ $\infty$

$$
{ }_{2} H_{r, s}^{S}=H_{r, s}^{*} .
$$

Благодарности. Авторы выражают благодарность рецензентам и редактору за полезные замечания.

\section{ЛитерАтУРА}

[1] C. Bennett, R. Sharpley, Interpolation of Operators, Academic Press, Boston, 1988. [2] J. Diestel, J. J. Uhl, Jr., Vector Measures, Amer. Math. Soc., Providence, RI, 1977. [3] Y. Jiao, Carleson measures and vector-valued BMO martingales, Prob. Theo. Rel. Fields (online first) (готовится к публикации). [4] P. D. Liu, Martingales and Geometry of Banach Spaces (in Chinese), Science Press, Beijing, 2007. [5] R-L. Long, Martingale Spaces and Inequalities, Peking University Press, Beijing, 1993. [6] T. Martínez, J. L. Torrea, Tohoku Math. J. (2), 52 (2000), 449-474. [7] G. Pisier, Israel J. Math., 20 (1975), 326-350. [8] Г. Пешкир, А. Н. Ширяев, УМН, 50:5 (1995), 3-62. [9] F. Weisz, Martingale Hardy Spaces and Their Applications in Fourier Analysis, Lecture Notes in Math., vol. 1568, Springer-Verlag, Berlin, 1994.

School of Mathematics Science and Computing Technology Central South University, Changsha, China

Laboratoire de Mathématiques, Université de France-Comté

Поступило в редакцию 31 октября 2008 г.

Besançon Cedex, France

e-mail: jjiao@univ-fcomte.fr

School of Mathematics and Statistics,

Wuhan University, China

e-mail: tma.math@whu.edu.cn

School of Mathematics and Statistics,

Wuhan University, China

e-mail: pdliu@whu.edu.cn

Заведующая редакцией и научный редактор Г. М. Цукерман

Сдано в набор 25.06.2010. Подписано к печати 27.07.2010. Формат 70×100/16 Печать офсетная. Усл. печ. л. 7,8. Усл. кр.-отт. 1,8 тыс. Бум. л. 3,0 Уч.-изд. л. 8,0. Тираж 226 экз. Заказ 435.

Учредитель: Российская академия наук

Адрес редакции: 117966 Москва, ГСП-1, ул. Губкина 8, комн. 624. Тел. 938-37-56

Издатель: Российская академия наук, Издательство «Наука», 117997 Москва, Профсоюзная, ул. 90

Отпечатано в ППП «Типография «Наука», 121099 Москва, Шубинский пер., 6 